\title{
HES5 promotes cellular proliferation of non-small cell lung cancer through STAT3 signaling
}

\author{
SHUDONG GU ${ }^{1-3 *}$, RUI ZHANG ${ }^{4 *}, \mathrm{JUN} \mathrm{GU}^{4}, \mathrm{XIA} \mathrm{LI}^{4}$, LITING $^{3}{ }^{3}$, JINGTING JIANG $^{1,2}$, \\ ZHEN XU ${ }^{3}$, SHUO WANG ${ }^{5}$, CUI SHI ${ }^{4}$, DAN PING WANG ${ }^{3}$ and CHANGPING WU ${ }^{1,2}$ \\ ${ }^{1}$ Department of Tumor Biological Treatment, The Third Affiliated Hospital of Soochow University, \\ Changzhou, Jiangsu 213003; ${ }^{2}$ Jiangsu Engineering Research Center for Tumor Immunotherapy, \\ Changzhou, Jiangsu 213003; Departments of ${ }^{3}$ Oncology, ${ }^{4}$ Respiratory, and ${ }^{5}$ Thoracic Surgery, \\ Affiliated Hospital of Nantong University, Nantong, Jiangsu 226001, P.R. China
}

Received June 10, 2016; Accepted October 31, 2016

DOI: $10.3892 /$ or.2016.5268

\begin{abstract}
HES5 is a transcription factor activated downstream of the Notch pathway and regulates cell differentiation in multiple tissues. Disruption of its normal expression has been associated with developmental diseases and cancer. But its role in non-small cell lung cancer (NSCLC) remains elusive. Western blot analysis and immunohistochemistry assays demonstrated that HES5 expression was frequently increased in NSCLC tumor tissues and cell lines. Moreover, immunohistochemistry analysis revealed that upregulation of HES5 expression was positively correlated with a more invasive tumor phenotype and poor prognosis. Immunoprecipitation assay indicated that HES5 directly interacted with STAT3. In addition, depletion of HES5 resulted in inhibited phosphorylation of STAT3 and decreased expression of the downstream genes. In vitro studies, using serum starvation-refeeding experiment and HES5-siRNA transfection assay demonstrated that HES5 expression promoted proliferation of NSCLC cells, while HES5 knockdown caused growth arrest of cell cycle at G0/G1 phase, decreased rate of colony formation and alleviated cellular apoptosis. Taken together, out data have delineated that HES5 might contribute to the proliferation of NSCLC by STAT3 signaling.
\end{abstract}

\section{Introduction}

Lung cancer is one of the leading causes of cancer-related death in the world (1). Human lung cancers can be classified into two major histological types, non-small cell lung cancer (NSCLC) and small cell lung cancer (SCLC), and the former

Correspondence to: Dr Changping Wu, Department of Tumor Biological Treatment, The Third Affiliated Hospital of Soochow University, Changzhou, Jiangsu 213003, P.R. China

E-mail: wcpjjt@163.com

*Contributed equally

Key words: HES5, proliferation, non-small cell lung cancer, STAT3 one accounts for more than $80 \%$ of all lung cancer cases (2). NSCLC is consisted of adenocarcinoma, squamous carcinoma, adenosquamous carcinoma and large cell carcinoma. Despite many advances achieved in the diagnosis and management of NSCLC, the mechanisms underlying the pathogenesis of NSCLC remain poorly understood and the overall 5-year survival rate was no more than $15 \%$ (3). The process of lung cancer is associated with alterations of both oncogenes and tumor suppressors, as well some cell cycle regulators related to cell proliferation and survival (4). Therefore, identifying new molecular targets and mechanisms can provide novel strategies for the diagnosis and treatment of NSCLC.

HES5 belongs to the basic helix-loop-helix (bHLH) superfamily and is a DNA-binding transcription factor. It is a downstream molecule and effector of mammalian Notch pathway, which is mainly expressed in epithelia in the process of embryogenesis or in neural stem cells (5). HES5 has an important role in regulating mammalian neuronal differentiation and the maintenance of neural stem cells (6) and positively regulates neuronal stem cell self-renewal (7). Recent studies have found that HES5 was involved in inducing cell differentiation or promoting the tumor cellular proliferation in many kinds of cancer, such as neuroblastoma cells, carcinoid tumor cells and breast cancer cell lines (8-10). This indicates that HES5 may be involved in the initiation and process of cancers. Furthermore, it was reported that HES5 could associate with JAK2 and STAT3, and promoted STAT3 phosphorylation and activation, which protected hepatocytes from apoptosis after I/R injury (11). Since many studies have revealed that JAK2-STAT3 could regulate cell proliferation and apoptosis in NSCLC $(12,13)$, HES5 may regulate the initiation and cell proliferation of NSCLC via STAT3 pathway.

In the present study, we investigated the role of HES5 in NSCLC's progression. Expression of HES5 in 8 paired tumor and adjacent non-tumor tissues were detected by western blot analysis. Immunohistochemistry (IHC) assay was performed in 114 NSCLC samples. Then, we also investigated the association of HES5 expression with clinical and pathological factors, as well as the prognostic implications. Immunoprecipitation assay and western blot analysis were used to detect the relation between HES5 and STAT3. Moreover, we explored the 
Table I. Expression of HES5 in 114 human lung NSCLC tissues.

\begin{tabular}{|c|c|c|c|c|c|}
\hline \multirow{2}{*}{$\begin{array}{l}\text { Clinicopathological } \\
\text { features }\end{array}$} & \multirow[b]{2}{*}{ Total } & \multicolumn{2}{|c|}{$\begin{array}{c}\text { HES5 } \\
\text { expression }\end{array}$} & \multirow[b]{2}{*}{ P-value } & \multirow[b]{2}{*}{$\chi^{2}$ value } \\
\hline & & Low & High & & \\
\hline \multicolumn{6}{|l|}{ Age (years) } \\
\hline$<60$ & 51 & 27 & 24 & 0.953 & 0.004 \\
\hline$\geq 60$ & 63 & 33 & 30 & & \\
\hline \multicolumn{6}{|l|}{ Gender } \\
\hline Female & 55 & 25 & 30 & 0.138 & 2,196 \\
\hline Male & 59 & 35 & 24 & & \\
\hline \multicolumn{6}{|l|}{ Tumor size $(\mathrm{cm})$} \\
\hline$<3$ & 50 & 38 & 12 & $0.046^{\mathrm{a}}$ & 19.508 \\
\hline$\geq 3$ & 64 & 22 & 42 & & \\
\hline \multicolumn{6}{|l|}{ Smoking status } \\
\hline Yes & 29 & 15 & 14 & 0.05 & 0.91 \\
\hline No & 85 & 45 & 40 & & \\
\hline \multicolumn{6}{|l|}{ Lymph node status } \\
\hline 0 & 73 & 47 & 26 & $0.033^{\mathrm{a}}$ & 11.244 \\
\hline$>0$ & 41 & 13 & 28 & & \\
\hline \multicolumn{6}{|l|}{ Clinical stage } \\
\hline I & 75 & 44 & 31 & $0.037^{\mathrm{a}}$ & 6.567 \\
\hline II & 25 & 13 & 12 & & \\
\hline III & 14 & 3 & 11 & & \\
\hline \multicolumn{6}{|l|}{$\begin{array}{l}\text { Histological } \\
\text { differentiation }\end{array}$} \\
\hline Well & 25 & 19 & 6 & $0.03^{\mathrm{a}}$ & 7.014 \\
\hline Moderate & 76 & 35 & 41 & & \\
\hline Poor & 13 & 6 & 7 & & \\
\hline \multicolumn{6}{|l|}{ Ki-67 expression } \\
\hline Low & 50 & 43 & 7 & $0.01^{\mathrm{a}}$ & 39.777 \\
\hline High & 64 & 17 & 47 & & \\
\hline
\end{tabular}

Statistical analyses were performed by Pearson's $\chi^{2}$ test. ${ }^{a} \mathrm{P}<0.05$ was considered significant.

potential involvement of HES5 in the regulation of cell cycle progression and cell proliferation in NSCLC cells using HES5 siRNA transfection.

\section{Materials and methods}

Patients and tissue samples. One hundred and fourteen lung cancer sections, 8 lung cancer tissue samples, and 8 normal tissue samples from patients who underwent surgery from the period of 2005 to 2009 at the Department of Pathology, Affiliated Hospital of Nantong University. The Affiliated Hospital of Nantong University Hospital provided formalinfixed and paraffin-embedded tissues for histopathological diagnosis and immunohistochemical study. The main clinical and pathological variables are shown in Table I. Besides, 8 paired tumor and adjacent non-tumor NSCLC fresh tissues were frozen in liquid nitrogen immediately and stored at $-80^{\circ} \mathrm{C}$ for western blot analysis.

Cell culture and transfection. The human NSCLC cell lines A549, H1299 and SPCA-1 were purchased from the China Academy of Science Cell Library (Beijing, China). All the cells were cultured in RPMI-1640 medium (Gibco-BRL, Grand Island, NY, USA) supplemented with $10 \%$ fetal bovine serum (FBS) at $37^{\circ} \mathrm{C}$ and $5 \% \mathrm{CO}_{2}$.

The HES5-siRNA and control-siRNA were chemically synthesized (Shanghai GenePharma, Co., Ltd., Shanghai, China). The HES5-specific siRNA target sequence was as follows: 5'-AAGGCTACTCGTGGTGCCT-3' named as siRNA\#1; 5'-AGGACTACAGCGAAGGCTA-3' named as siRNA\#2; and 5'-TGTCAGCTACCTGAAGCAC-3' named as SiRNA\#3.

A549 cells were grown in dishes until they reached $70 \%$ confluence. The medium was replaced $6 \mathrm{~h}$ later with fresh medium for transfection. A549 cells were transfected with HES5-siRNA or control-siRNA according to the manufacturer's instructions. Cells were collected for western blot analysis, CCK-8 and flow cytometry assays after transfection for $48 \mathrm{~h}$ (14).

Western blot analysis. Tissue and cell protein were collected with two lysis buffers containing $50 \mathrm{mM}$ Tris- $\mathrm{HCl}, \mathrm{pH} 7.5$; $150 \mathrm{mM} \mathrm{NaCl} ; 0.1 \%$ NP-40; 5 mM EDTA; 60 mMb glycerophosphate; $0.1 \mathrm{Mm}$ sodium orthovanadate; $0.1 \mathrm{mM} \mathrm{NaF}$; and complete protease inhibitor cocktail (Roche Diagnostics, Indianapolis, IN, USA) and then incubated for $20 \mathrm{~min}$ at $4^{\circ} \mathrm{C}$ while rocking. Lysates were collected after centrifugation (15 min at $12,000 \mathrm{rpm}, 4^{\circ} \mathrm{C}$ ). Protein concentrations were measured with a Bio-Rad protein assay (Bio-Rad Laboratories, Hercules, CA, USA). Then, the same total protein was separated by SDS-PAGE and transferred to a polyvinylidene fluoride (PVDF) membrane (Immobilon; Millipore, Billerica, MA, USA). The membranes were first blocked with $5 \%$ non-fat milk in TBST $(150 \mathrm{mM} \mathrm{NaCl}, 20 \mathrm{mM}$ Tris and $0.05 \%$ Tween20 ), and the membranes were washed with TBST three times after $2 \mathrm{~h}$ at room temperature and then incubated overnight with the primary antibodies. Then, LumiGLO reagent and peroxide (Cell Signaling Technology, Danvers, MA, USA) was used as the secondary antibody. The band was then detected by enhanced chemiluminescence (ECL) detection systems (Pierce, Rockford, IL, USA). The band intensity was measured by an ImageJ analysis system (Wayne Rasband; National Institutes of Health, Bethesda, MD, USA) (15).

Antibodies. The antibodies used in the western blot analysis included anti-HES5 (anti-rabbit, 1:500; Santa Cruz Biotechnology, Santa Cruz, CA, USA), anti-STAT3 (antimouse, 1:500; Santa Cruz Biotechnology), anti-p-STAT3 (anti-rabbit, 1:1,000; Abcam), anti-PCNA (anti-mouse, 1:1,000; Santa Cruz Biotechnology) and anti-GAPDH (anti-rabbit, 1:3,000; Sigma-Aldrich).

Immunohistochemistry. Surgically excised tissues were fixed with $10 \%$ formalin and embedded in paraffin, and $4-\mu \mathrm{m}$-thick specimen sections were prepared on glass slides. The sections 
were deparaffinized in xylene and rehydrated with graded alcohol, and then, antigen retrieval was performed by heating to $121^{\circ} \mathrm{C}$ for $3 \mathrm{~min}$ in $10 \mathrm{mmol} / \mathrm{l}$ citrate buffer ( $\mathrm{pH} \mathrm{6.0)}$ with an autoclave. Thereafter, endogenous peroxidase activity was blocked by soaking in $0.3 \%$ hydrogen peroxide for $20 \mathrm{~min}$. After rinsing in phosphate-buffered saline (PBS) ( $\mathrm{pH} 7.2)$, the sections were then incubated with anti-HES5 antibody (diluted 1:100; Santa Cruz Biotechnology) for $2 \mathrm{~h}$ at room temperature, and anti-Ki-67 antibody (diluted 1:400; Santa Cruz Biotechnology) for $2 \mathrm{~h}$ at room temperature. All slides were processed using the peroxidase-antiperoxidase method (Dako, Hamburg, Germany). After being washed in PBS, the peroxidase reaction was visualized by incubating the sections with DAB $(0.1 \%$ phosphate buffer solution, $0.02 \%$ diaminobenzidine tetrahydrochloride and $3 \% \mathrm{H}_{2} \mathrm{O}_{2}$ ). After rinsing in water, the sections were counterstained with hematoxylin, dehydrated and coverslipped.

Immunohistochemical evaluation. All of the immunostained sections were evaluated in a blinded manner without knowledge of the clinical and pathological parameters of the patients. For assessment of HES5 and Ki-67, five views were chosen per slide, and at least 1,000 cells were counted per view at high power fields. In more than one half of the samples, staining was repeated three times to avoid technical errors, and a consensus was achieved. Three independent pathologists evaluated the immunostaining results. For statistical analysis of HES5 stain, each slide was evaluated using a semi-quantitative scoring system for both the intensity of the stain and the percentage of positive malignant cells (16). The intensity of staining was coded as follows: 0 (negative or poor staining), 1 (moderate staining), and 2 (strong staining). The percentage of cells was scored as follows: low-expression group $(<50 \%)$ score 1, moderate-expression group (50-75\%) 2 , and high expression group $(>75 \%)$ score 3 . Then, we multiplied the two scores and divided patients into two groups according to the average scores (3): high-expression group (>3) and low expression group ( $\leq 3)$. In statistical analysis of Ki-67 stain, $50 \%$ of malignant cells showing positive stain was used as a cut-off value to distinguish tumors with a low $(<50 \%)$ or high $(\geq 50 \%)$ level of expression (17).

Cell cycle analysis. A serum starvation and refeeding process was used to imitate the cell cycle. First, we used RPMI-1640 medium without FBS to incubate A549 cells for $72 \mathrm{~h}$ to synchronize cells, which was then changed into complete medium. Then, cells were fixed in $70 \%$ ethanol for $1 \mathrm{~h}$ at $4^{\circ} \mathrm{C}$ and incubated with $1 \mathrm{mg} / \mathrm{ml}$ RNaseA for $30 \mathrm{~min}$ at $37^{\circ} \mathrm{C}$. Subsequently, cells were stained with propidium iodide (PI, $50 \mu \mathrm{g} / \mathrm{ml}$ PI) (Becton Dickinson, San Jose, CA) in PBS and $0.5 \%$ Triton X-100, and analyzed using a Becton Dickinson flow cytometer BD FACScan (Becton Dickinson) as well as CellQuest acquisition and analysis programs.

Cell Counting kit-8 assays. Commercial Cell Counting kit-8 (CCK-8) assays (Dojindo Laboratories, Kumamoto, Japan) were performed to evaluate cell proliferation. A549 cells transfected with HES5-siRNA and control-siRNA were seeded onto 96-well cell culture cluster plates (Corning, Inc., Corning NY, USA) at a concentration of $2 \times 10^{4}$ cells/well in volumes of $100 \mu \mathrm{l}$ and grown overnight. CCK-8 reagents (Dojindo Laboratories) were added to each well under different timepoints, and the wells were incubated for an additional $2 \mathrm{~h}$ at $37^{\circ} \mathrm{C}$ in the dark. The absorbency was measured at a test wavelength of $450 \mathrm{~nm}$ and a reference wavelength of $650 \mathrm{~nm}$ with a microplate reader (Bio-Rad Laboratories). The experiments were repeated at least three times.

Statistical analysis. The SPSS 19.0 statistical program was used for statistical analysis. The HES5 expression and clinicopathological features were analyzed by the Chi-square $\left(\chi^{2}\right)$ test. For analysis of survival data, Kaplan-Meier curves were constructed and log-rank test was performed. Multivariate analysis was performed using Cox's proportional hazards model. The risk ratio and its $95 \%$ confidence interval were recorded for each marker. $\mathrm{P}<0.05$ was considered statistically significant for all of the analysis. The values were expressed as mean \pm SEM (18). Each experiment consisted of at least three replicates per condition.

\section{Results}

HES5 was increased in NSCLC tissues. To explore whether abnormal expression of HES5 is associated with the progression of NSCLC, first, we investigated the expression of HES5 between eight paired NSCLC tissues and the adjacent nontumorous tissues by western blotting. As shown in Fig. 1A, HES5 was significantly upregulated in most NSCLC tissues compared to the adjacent non-tumor tissues. Moreover, we examined the expression profile of HES5 in three human NSCLC cell lines, SPCA1, H1299 and A549. As expected, HES5 was highly expressed in A549 cell line, while there was no expression in the other two cell lines (Fig. 1C). To further investigate the expression of HES5 in clinicopathological specimens, 114 samples from patients with NSCLC were analyzed using immunohistochemical assay. We found immunoreactivity of HES5 was seen mainly in the nucleus (Fig. 2). While $\mathrm{Ki}-67$, which is a cell proliferation index, was also expressed predominantly in the nucleus (Fig. 2). These findings together revealed that HES5 was highly expressed in NSCLC tissues.

HES5 expression is correlated with $\mathrm{Ki}-67$ in NSCLC - relationship to clinicopathological variables. Next, we evaluated the clinicopathological significance of HES5 expression and the physiological or pathologicalal association between HES5 and $\mathrm{Ki}-67$ in clinicopathological variables of NSCLC. As shown in Table I, we found that the expression of HES5 was significantly related with tumor size $(\mathrm{P}=0.046)$, lymph node metastasis $(\mathrm{P}=0.033)$, clinical stage $(\mathrm{P}=0.037)$, histological differentiation $(\mathrm{P}=0.03)$ and $\mathrm{Ki}-67$ expression $(\mathrm{P}=0.01)$, while there was no correlation with other prognostic factors such as age $(\mathrm{P}=0.953)$ and gender $(\mathrm{P}=0.138)$. Furthermore, we found that there was a positive relation between the expression HES5 expression and Ki-67 using Spearman's rank correlation test $\left(\mathrm{P}<0.01, \mathrm{r}^{2}=0.317\right.$; Fig. 3). Taken together, upregulated expression of HES5 could be a strong determinant of poor prognosis in NSCLC.

High expression of HES5 correlates with poor survival of NSCLC patients. We used Kaplan-Meier analysis to calcu- 
A



GAPDH

$\mathrm{B}$

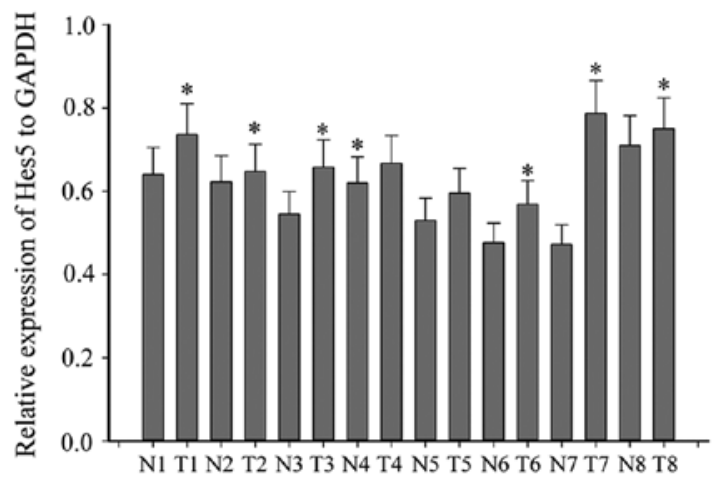

$\mathrm{C}$

SPCA-1 H1299 A549

HES5

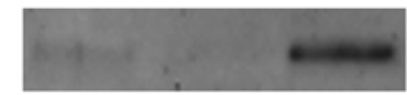

GAPDH

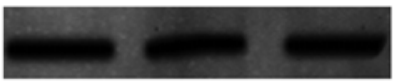

$\mathrm{D}$

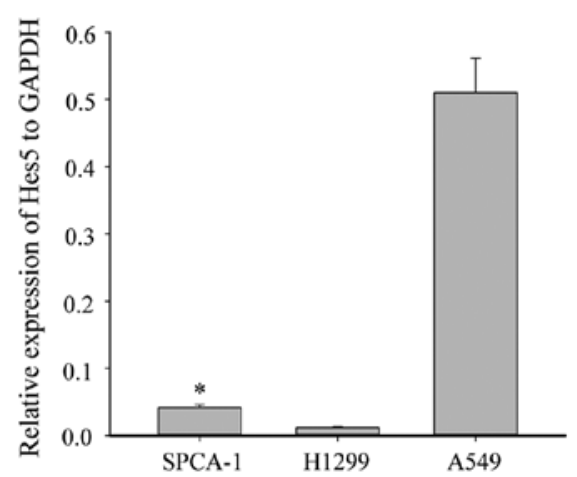

Figure 1. HES5 was increased in lung cancer tissues compared with adjacent non-tumorous tissues. (A) The expression of HES5 was determined in 8 paired samples of NSCLC tumor tissues (T) and adjacent non-tumor tissues (N) using western blot assay. GAPDH was used as a loading control. (B) The bar chart demonstrated the relative expression levels of HES5 and PCNA to GAPDH by densitometry. The data are mean \pm SEM ("P $<0.05$, compared with adjacent tumor tissues). The same experiment was repeated at least 3 time. (C) HES5 was expressed in three NSCLC cells. The expression of HES5 was significantly upregulated and the relative expression levels were shown by density photometry. The same experiment was repeated at least three times.
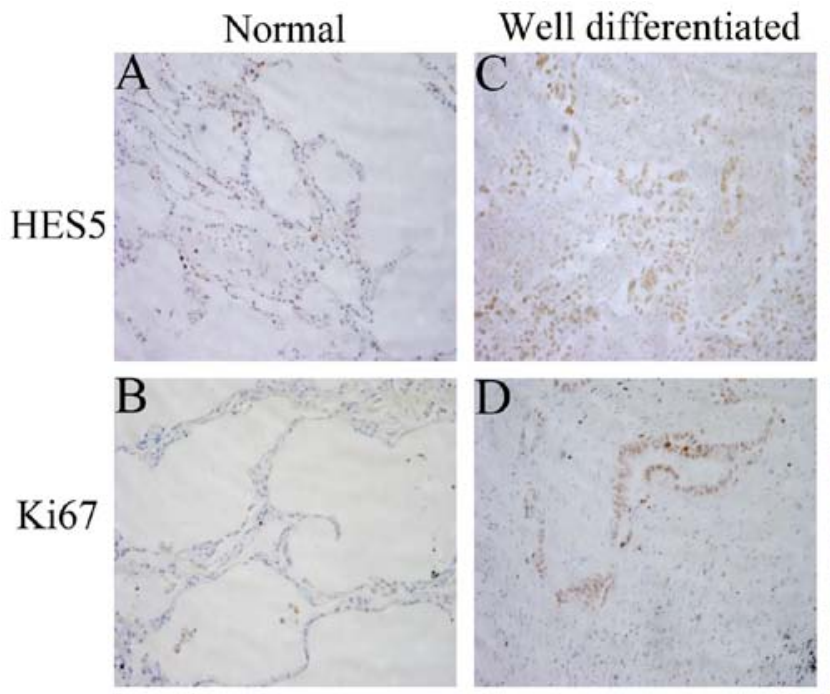
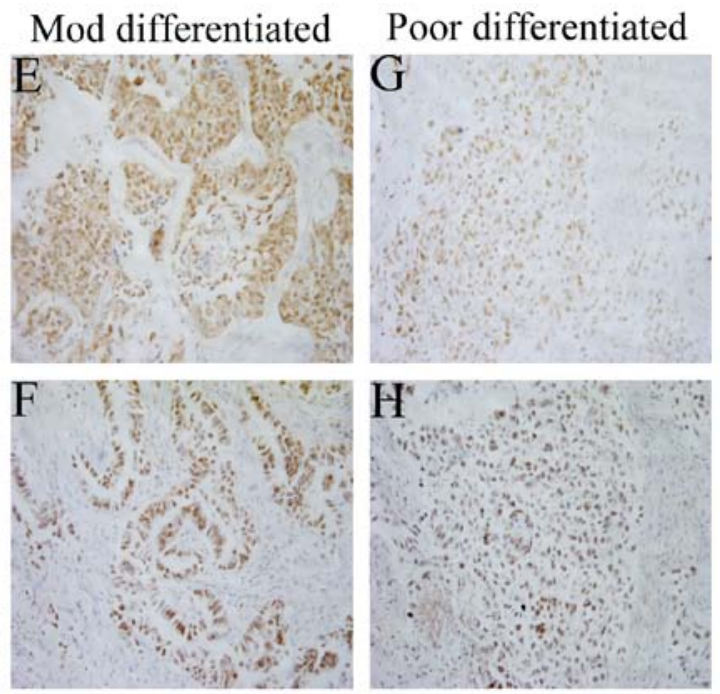

Figure 2. Immunohistochemical stain of HES5 and Ki-67 in NSCLC tissues. Paraffin embedded tissue sections were stained for HES5 and Ki-67. HES5 and $\mathrm{Ki}$-67 expression in adjacent non-tumor tissues (A and B), well differentiated (C and D), moderate differentiated (E and $\mathrm{F})$ and poorly differentiated $(\mathrm{G}$ and $\mathrm{H})$ NSCLC tissues (magnification, $\mathrm{x} 200$ ).

late the association between the expression of HES5 and patients' survival (Fig. 4). The survival curves showed that NSCLC patients with high HES5 expression had significantly decreased overall survival, compared with those with low HES5 expression. Moreover, Cox proportional hazards model indicated that HES5 expression ( $\mathrm{P}=0.027)$, as well as Ki-67
$(\mathrm{P}=0.018)$ and histological differentiation $(\mathrm{P}=0.048)$, were independent prognostic indicators for overall survival of the patients (Table II).

Expression of HES5 promotes proliferation of NSCLC cells. Based on the the fact that HES5 expression was positively 


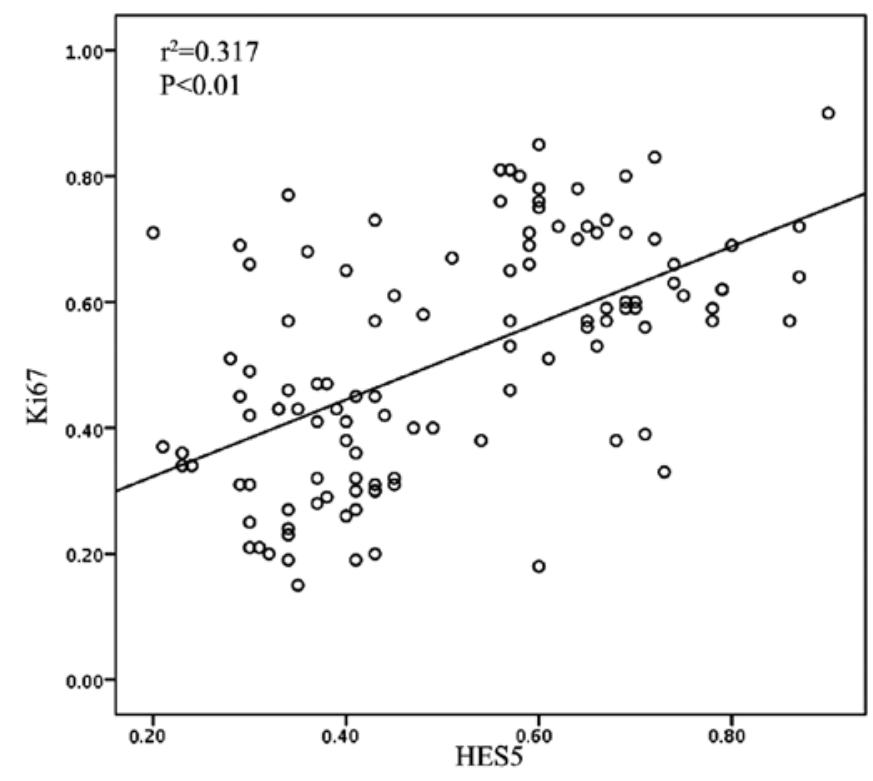

Figure 3. Correlation between HES5 and Ki-67 expression in NSCLC samples. The correlation between HES5 and Ki-67 expression in NSCLC was further evaluated by Spearman's rank correlation test $(\mathrm{P}<0.05)$.

Table II. Contribution of various potential prognostic factors to survival by Cox regression analysis in 114 NSCLC specimens.

\begin{tabular}{lccc}
\hline & Hazard ratio & $95.0 \%$ CI & P-value \\
\hline Age & 1.245 & $0.703-2.206$ & 0.453 \\
Gender & 0.542 & $0.532-2.705$ & 0.660 \\
Clinical stage & 1.055 & $0.686-1.623$ & 0.406 \\
Tumor size & 1.448 & $0.653-3.212$ & 0.363 \\
Histological & & & \\
differentiation & 0.587 & $0.345-0.996$ & $0.048^{\mathrm{a}}$ \\
Lymph node status & 0.987 & $0.534-1.822$ & 0.966 \\
Ki-67 expression & 1.241 & $0.326-0.901$ & $0.018^{\mathrm{a}}$ \\
HES5 expression & 1.922 & $1.077-3.430$ & $0.027^{\mathrm{a}}$ \\
\hline
\end{tabular}

${ }^{\text {a }}$ Statistical analyses were performed by the Cox regression analysis and $\mathrm{P}<0.05$ was considered significant. $\mathrm{CI}$, confidence interval.

correlated with the expression of Ki-67 and higher histological grade, we speculated that HES5 might play a role in cell cycle progression of NSCLC cells. To verify this hypothesis, we chose A549 cells for the serum starvation and refeeding process. A549 cells were serum starved for $72 \mathrm{~h}$ and then recovered by serum refeeding. We analyzed the cell cycle progression after serum deprivation for $48 \mathrm{~h}$ using flow cytometry. As shown in Fig. 5A and B, A549 cells were arrested in G1 phase. Upon serum addition, A549 cells were released from the G0/ G1 phase and gradually entered into $\mathrm{S}$ and $\mathrm{G} 2 / \mathrm{M}$ phases. To further confirm the results, western blot assays were performed to analyze proliferating cell nuclear antigen (PCNA) and the expression of HES5. We found that the expression of PCNA and cell cycle regulator cyclin D1 were increased after serum stimulation in A549 cells. The protein level of HES5 was also upregulated (Fig. 5C and D). Thus, this result suggested that

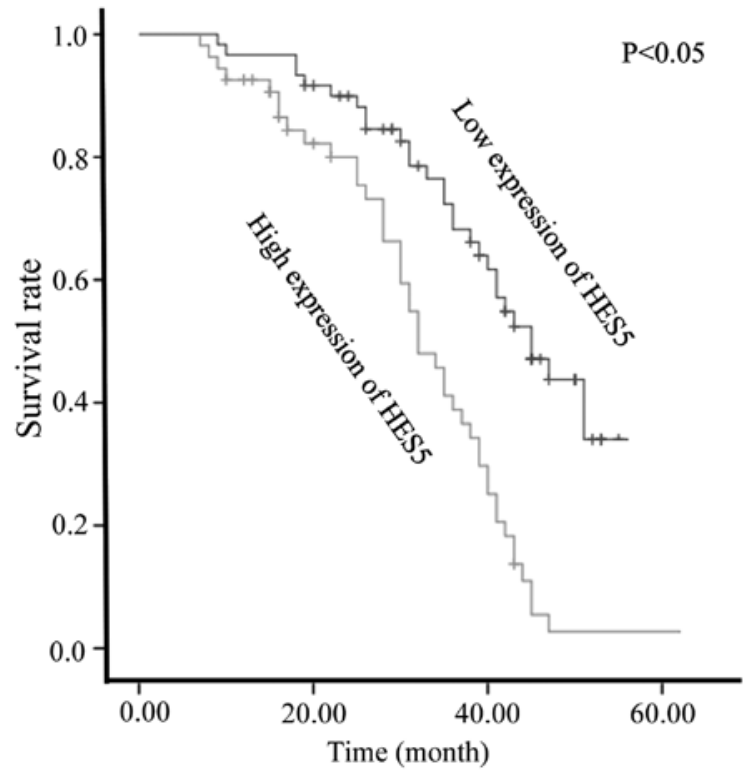

Figure 4. Kaplan-Meier survival analysis of HES5 expression status. Cumulative overall survival differences between patients with high and low level of HES5 protein expression. Patients within the high expression group show worse overall survival.

HES5 might have a function as a positive regulator of NSCLC cells in a cell cycle-dependent manner.

HES5 interacts with STAT3 and affects activation of STAT3. Taken together, the results above demonstrated that HES5 was obviously higher expressed in NSCLC cells. Cross-talk has been reported between HES5 and STAT3 in hepatocytes and hepatocellular carcinoma, which provided a reason to research the mechanism of HES5 involved in NSCLC. There are many studies suggesting that STAT3 was closely related with the proliferation and growth of NSCLC. As a result, we performed immunoprecipitation to further investigate the mechanism of HES5 on cellular proliferation. Co-immunoprecipitation experiments were on non-transfected A549 cell lysates with anti-HES5 or anti-STAT3 antibody. As shown in Fig. 6A and B, HES5 could co-immunoprecipitate with the anti-STAT3 antibody, but not in control precipitation, indicating a naturally occurring interaction between endogenous HES5 and STAT3 in vivo, and vice versa. Furthermore, we explored whether the HES5/STAT3 interaction can be detected exogenously. We transfected A549 cells with HA-HES5 and anti-HA was used to precipitate STAT3. It was shown that STAT3 co-immunoprecipitated specifically with anti-HA antibody, which demonstrated that HES5 could interact with STAT3 in vitro (Fig. 6C). To further investigate the role of HES5 in cellular proliferation, we analyzed HES5 in A549 cells using lentivirus-mediated RNA interference. We transiently transfected A549 cells with HES5-siRNA\#1, HES5-siRNA\#2, HES5-siRNA\#3 or control siRNA. After $36 \mathrm{~h}$, western blot analysis was used to evaluate the efficiency of transfection. We found that HES5 protein levels decreased most significantly in A549 cells infected with HES5-siRNA\#2, which compared with cells treated with other si-HES5 (Fig. 6D). Thus, we used HES5-siRNA\#2 to perform the following experiments. To evaluate whether loss of STAT3 activation 
A

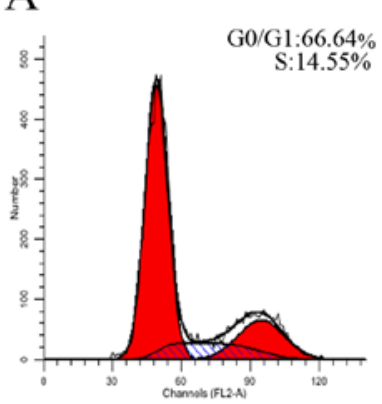

$\mathrm{S} 72 \mathrm{~h}$

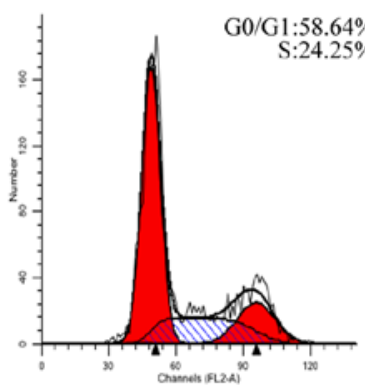

$\mathrm{R} 8 \mathrm{~h}$

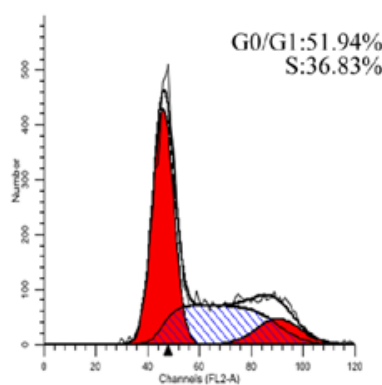

R24h

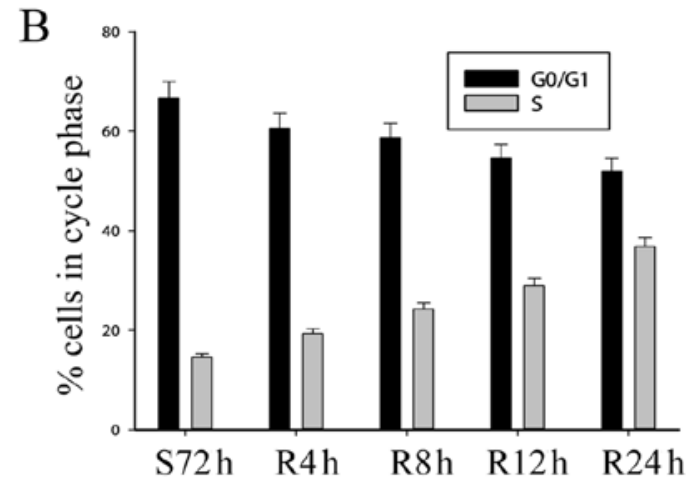

C

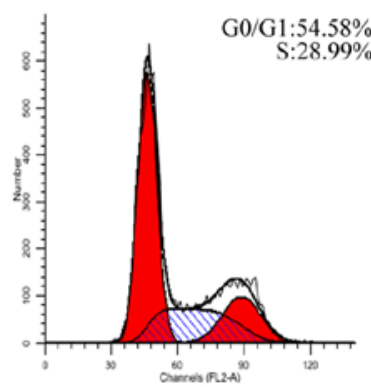

$\mathrm{R} 12 \mathrm{~h}$

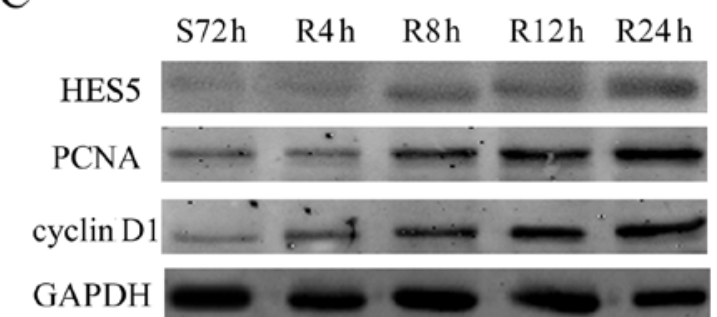

D

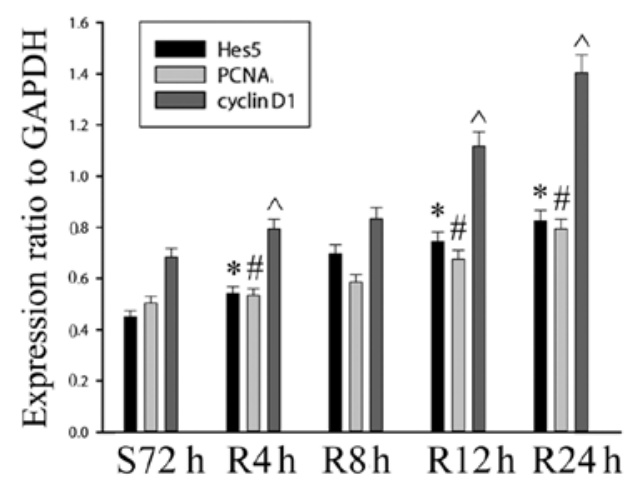

Figure 5. Expression of HES5 promotes proliferation of NSCLC cells. (A and B) Cells were synchronized at G0/G1 and progressed into the cell cycle when serum was added for S72 h, R6 h, R12 h, R24 h and R48 h. The experiment was conducted by flow cytometry. (C and D) The S72 h A549 cells were released by refeeding with serum, and cell lysates were prepared and analyzed by western blot analysis using antibodies against HES5, cyclin D1, PCNA and GAPDH (loading control). The bar chart demonstrates the ratio of HES5, cyclin D1 and PCNA to GAPDH by densitometry. The data are means \pm SEM. ${ }^{*, \pm .}{ }^{\circ} \mathrm{P}<0.05$, compared with control cells serum starved for $48 \mathrm{~h}$ (S72 h). S, serum starvation; R, serum release.

was involved in the effects induced by HES5 knockdown, we detected STAT3 and the expression of its target genes. We observed that knockdown of HES5 inhibited the activation of STAT3 and decreased the expression of cyclin D1 and Bcl-xL in A549 cells transfected with HES5-siRNA\#2 (Fig. 6E). Thus, these results suggest that HES5 is responsible for tumor oncogenicity and regulate proliferation in NSCLC via STAT3 pathway.

Knockdown of HES5 inhibits cell proliferation in NSCLC cells. Next, we investigated the role of HES5 on cell proliferation in NSCLC. We transfected A549 cells with control-siRNA and HES5-siRNA\#2, and the flow cytometric analyses of the cell cycle showed an increase of cell number in the G0/G1 phase from 60.85 to $68.44 \%$ and a decrease of cell number in the S phase from 15.53 to $10.59 \%$ (Fig. 7A and B). It indicates that downregulation of HES5 could slow down the NSCLC cell cycle. In addition, a CCK-8 assay was used to confirm the effect of HES5. Knockdown of HES5 can decrease the cell proliferation (Fig. 7C). By colony formation assay, we found that the rate of colony formation was significantly attenuated in knockdown of HES5 (Fig. 7D). Furthermore, the Annexin V-FITC/7-ADD assay also showed significant increase of cell death after HES5 knockdown (Fig. 7E). In conclusion, these results suggested that knockdown of HES5 might inhibit G0/G1-S transition and delay the proliferation of A549 cells.

\section{Discussion}

The initiation and progression of NSCLC is comprehensive, where complex alterations of oncogenes and tumor suppressor genes are involved. Despite significant progress in diagnostic and therapeutic strategy, the prognosis of NSCLC patients remained unsatisfactory due to high incidence of tumor recurrence, invasion and metastasis. Therefore, it is urgent to identify novel therapeutic targets and develop new anticancer therapies such as molecular-targeted drugs or antibodies. 
A

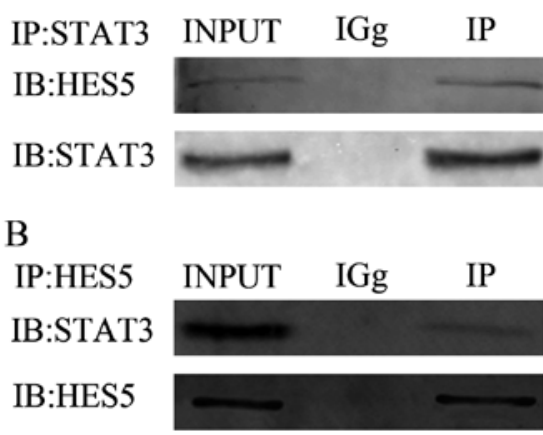

C

IP:HA

HA HA-HES5

IB:STAT3

IB:HES5

D


HES5
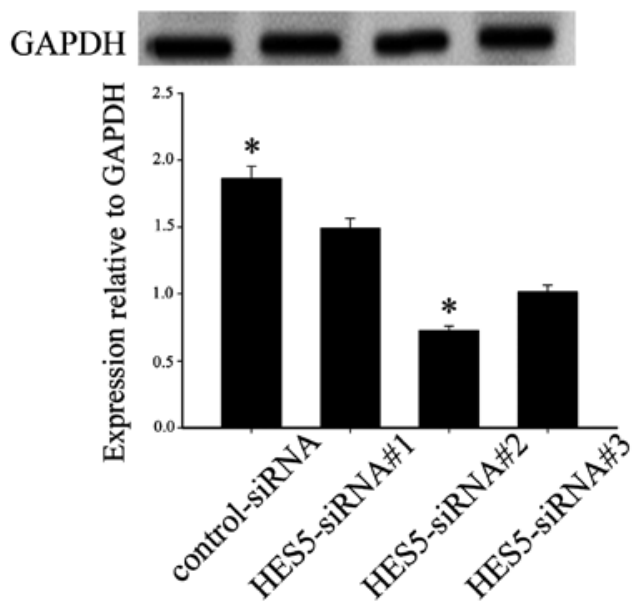

E



STAT3

Cyclin D1

Bcl-xL

GAPDH
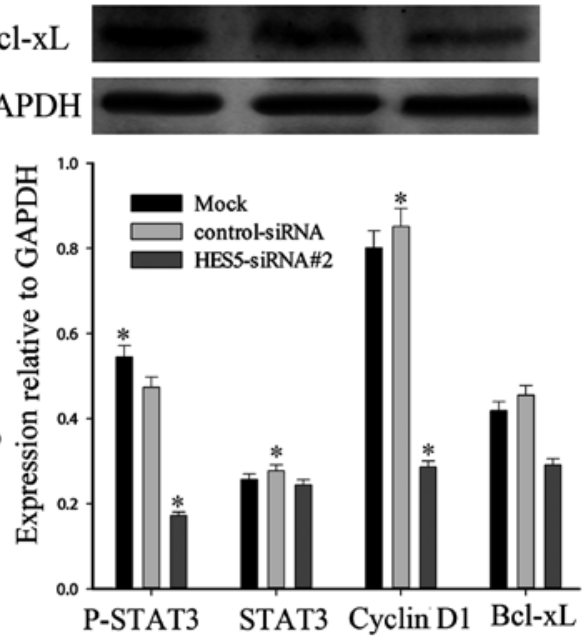

Figure 6. The interaction of HES5 with STAT3 and effects of HES5 knockdown on the expression of STAT3 and STAT3 downstream targets. (A and B) Co-immunoprecipitation experiments were conducted on non-transfected A549 cell lysates with anti-HES5 or anti-STAT3 antibody. Proteins in the cellular lysates and immunoprecipitated (IP) proteins were subsequently analyzed by western blotting (IB) the indicated antibodies. (C) A549 cells were transfected with HA-HES5 as indicated. Proteins in the cellular lysates and immunoprecipitated (IP) proteins were subsequently analyzed by western blotting (IB) the indicated antibodies. (D) Cellular proteins were analyzed by western blotting in NSCLC cells transfected with control siRNA and HES5-siRNA\#2 groups with antibodies specific for pSTAT3, STAT3, cyclin D1, Bcl-xL and GAPDH. (E) The ratio of colorimetric density of STAT3 and downstream genes. GAPDH served as loading control. The data are means \pm SEM. ${ }^{*} \mathrm{P}<0.05$ compared with the control. The results are for three independent experiments.

HES5, together with HES1, is an important transcription factor that involved in neural stem cells. HES1 has similar sequence with HES5, which suggests that they are probably functionally related genes. Previous studies showed that HES1 is involved in oncogenesis. HES1 is highly expressed in many cancer types, including colorectal cancer cells, cervical carcinoma cells and colon cancer (19-21). A study in human lung cancers also showed that HES1 expression was at abundant level in several non-small cell lung cancer cell lines without neuroendocrine features (22), but the roles of HES5 in NSCLC remain unknown.
In the present study, we confirmed that HES5 might be an important regulator in cell proliferation of NSCLC. Firstly, we analyzed the HES5 expression in NSCLC tissues and the cell lines using western blot analysis. The results revealed that HES5 was upregulated in NSCLC tumor tissues and NSCLC cell line compared with adjacent non-tumor ones (Fig. 1). Secondly, immunohistochemistry analysis of 114 NSCLC samples were performed and showed that HES5 expression was associated with tumor size, histological differentiation, clinical stage, lymph node status and Ki-67 expression (Table I). Ki-67 is a useful marker of tumor proliferative activity and only 
A

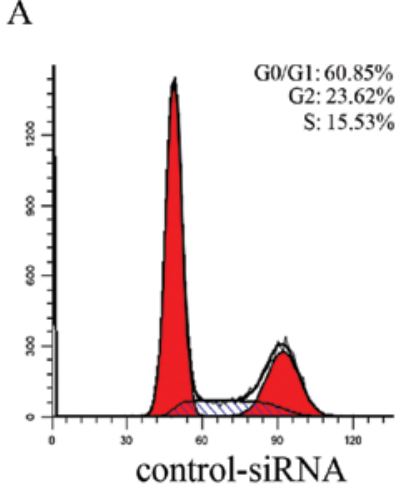

$\mathrm{C}$

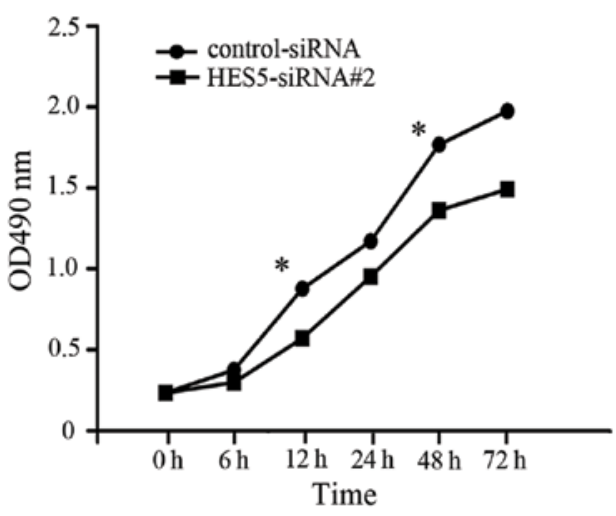

$\mathrm{E}$

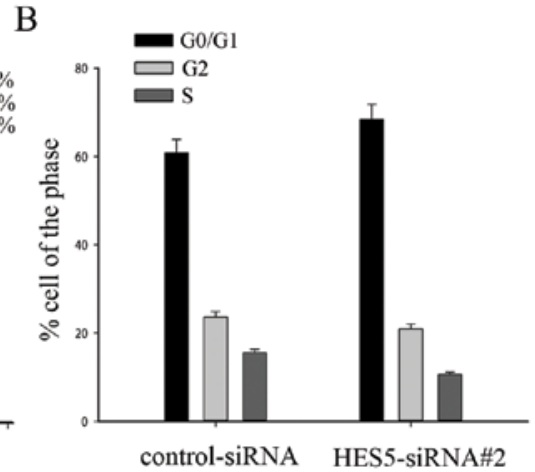

$\mathrm{D}$
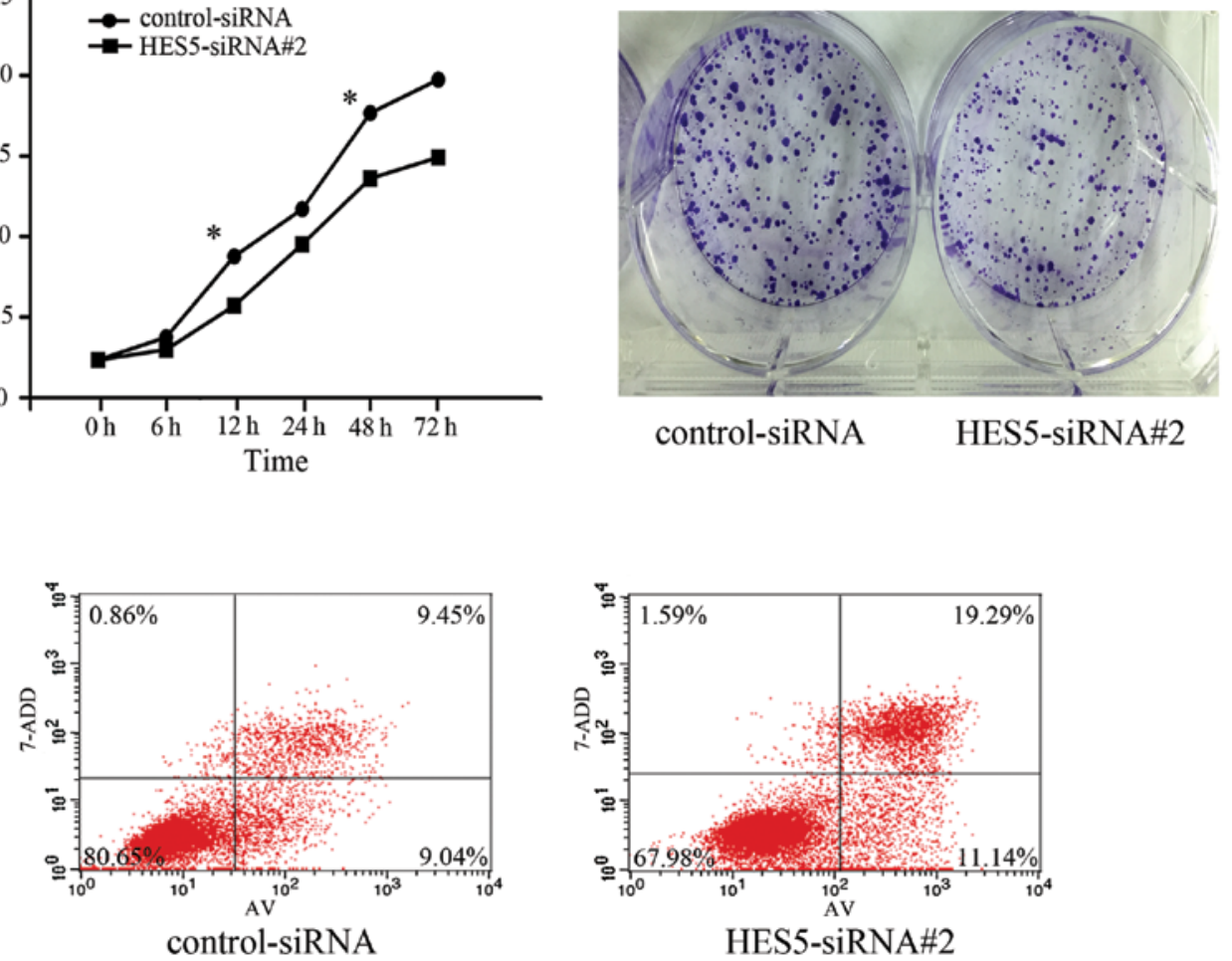

Figure 7. Knockdown of HES5 inhibits cell proliferation. (A) Cell cycle analysis was performed after knockdown of HES5 by HES5-siRNA\#2 in A549 cells. (B) The ratio of colorimetric density of the cell cycle. (C) CCK-8 assay was measured by cell proliferation. A549 cells treated with HES5-siRNA\#2 revealed significantly weakened proliferation. Absorbance was used to examine the control siRNA- and HES5-siRNA\#2-treated A549 cells. (D) Colony formation analysis of control siRNA- and HES5-siRNA\#2 transfected A549 cells. (E) Annexin V-FITC/7-ADD assay displayed a significant increase of cell death after HES5 knockdown. The data are means \pm SEM. ${ }^{*} \mathrm{P}<0.05$ compared with the control. The results are from three independent experiments.

expressed during the active phases of the cell cycle $(23,24)$. In addition, multivariate analysis indicated that HES5 could be an independent prognostic factor for the survival of NSCLC patients (Table II). Furthermore, Kaplan-Meier analysis showed that overexpression of HES5 predicted poor survival (Fig. 4). We found that HES5 expression was positively correlated with cell proliferation by serum starvation and release assay (Fig. 5). Knockdown of HES5 resulted in decreased rate of cell growth, colony formation and alleviated cellular apoptosis. In addition, we performed cell cycle analysis in NSCLC cells, and we found that HES5 expression was increasingly upregulated during $\mathrm{G} 1$ to $\mathrm{S}$ phase, while the proportion of cells in S phase was decreased in cells low expressed of HES5 (Fig. 7). Therefore, HES5 may be involved in the process of NSCLC tumorigenesis.
Previous studies revealed that HES5 proteins associated with STAT3 and JAK2, and facilitated complex formation between STAT3 and JAK2, thus, promoting STAT3 phosphorylation and activation $(11,25)$. We demonstrated that HES5 interacted with STAT3 in NSCLC cells, and knockdown of HES5 could inhibit the activity of STAT3 and decrease the expression of the downstream targets (Fig. 6). This suggested that HES5 might affect the proliferation through STAT3 pathway. However, further studies are needed to clarify the molecular mechanisms of HES5 in NSCLC pathogenesis.

In summary, all these results showed that HES5 was upregulated in NSCLC and promoted cell proliferation process through the activation of STAT3. Therefore, HES5 might be a novel molecular target for the diagnosis and therapy of NSCLC. 


\section{Acknowledgements}

The present study was supported by the National Natural Science Foundation of China (no. 81472185).

\section{References}

1. Wang J, Wei H, Zhao B, Li M, Lv W, Lv L, Song B and Lv S: The reverse effect of $X$-ray irradiation on acquired gefitinib resistance in non-small cell lung cancer cell line NCI-H1975 in vitro. J Mol Histol 45: 641-652, 2014.

2. Siegel R, Ma J, Zou Z and Jemal A: Cancer statistics, 2014. CA Cancer J Clin 64: 9-29, 2014.

3. Liu SV and Giaccone G: Lung cancer in 2013: Refining standard practice and admitting uncertainty. Nat Rev Clin Oncol 11: 69-70, 2014.

4. Osada $\mathrm{H}$ and Takahashi $\mathrm{T}$ : Genetic alterations of multiple tumor suppressors and oncogenes in the carcinogenesis and progression of lung cancer. Oncogene 21: 7421-7434, 2002.

5. Ohtsuka T, Sakamoto M, Guillemot F and Kageyama R: Roles of the basic helix-loop-helix genes Hes1 and Hes5 in expansion of neural stem cells of the developing brain. J Biol Chem 276 30467-30474, 2001

6. Ohtsuka T, Ishibashi M, Gradwohl G, Nakanishi S, Guillemot F and Kageyama R: Hes1 and Hes5 as notch effectors in mammalian neuronal differentiation. EMBO J 18: 2196-2207, 1999.

7. Hatakeyama $\mathbf{J}$ and Kageyama R: Notch1 expression is spatiotemporally correlated with neurogenesis and negatively regulated by Notch1-independent Hes genes in the developing nervous system. Cereb Cortex 16 (Suppl 1): i132-i137, 2006.

8. Axelson $\mathrm{H}$ : The Notch signaling cascade in neuroblastoma: Role of the basic helix-loop-helix proteins HASH-1 and HES-1. Cancer Lett 204: 171-178, 2004.

9. Kunnimalaiyaan M, Yan S, Wong F, Zhang YW and Chen $\mathrm{H}$ : Hairy enhancer of Split-1 (HES-1), a Notch1 effector, inhibits the growth of carcinoid tumor cells. Surgery 138: 1137-1142, discussion 1142,2005

10. Ström A, Arai N, Leers J and Gustafsson JA: The hairy and enhancer of Split homologue-1 (HES-1) mediates the proliferative effect of 17 beta-estradiol on breast cancer cell lines. Oncogene 19: 5951-5953, 2000.

11. Yu HC, Qin HY, He F, Wang L, Fu W, Liu D, Guo FC, Liang L, Dou KF and Han H: Canonical notch pathway protects hepatocytes from ischemia/reperfusion injury in mice by repressing reactive oxygen species production through JAK2/STAT3 signaling. Hepatology 54: 979-988, 2011.

12. Haura EB, Zheng Z, Song L, Cantor A and Bepler G: Activated epidermal growth factor receptor-Stat-3 signaling promotes tumor survival in vivo in non-small cell lung cancer. Clin Cancer Res 11: 8288-8294, 2005.

13. Malanga D, De Marco C, Guerriero I, Colelli F, Rinaldo N, Scrima M, Mirante T, De Vitis C, Zoppoli P, Ceccarelli M, et al: The Akt1/IL-6/STAT3 pathway regulates growth of lung tumor initiating cells. Oncotarget 6: 42667-42686, 2015.
14. Xue Q, Zhou Y, Wan C, Lv L, Chen B, Cao X, Ju G, Huang Y, Ni R and Mao G: Epithelial membrane protein 3 is frequently shown as promoter methylation and functions as a tumor suppressor gene in non-small cell lung cancer. Exp Mol Pathol 95: 313-318, 2013.

15. Ji L, Ni T, Shen Y, Xue Q, Liu Y, Chen B, Cui X, Lv L, Yu X, Cui Y, et al: Transformer $2 \beta$ (Tra2 $\beta / S F R S 10)$ positively regulates the progression of NSCLC via promoting cell proliferation. J Mol Histol 45: 573-582, 2014

16. Ni T, Mao G, Xue Q, Liu Y, Chen B, Cui X, Lv L, Jia L, Wang Y and Ji L: Upregulated expression of ILF2 in non-small cell lung cancer is associated with tumor cell proliferation and poor prognosis. J Mol Histol 46: 325-335, 2015.

17. Chen J, Gu J, Feng J, Liu Y, Xue Q, Ni T, Wang Z, Jia L, Mao G and Ji L: TAB3 overexpression promotes cell proliferation in non-small cell lung cancer and mediates chemoresistance to CDDP in A549 cells via the NF-kappaB pathway. Tumour Biol 37: 3851-3861, 2015.

18. Xue Q, Lv L, Wan C, Chen B, Li M, Ni T, Liu Y, Liu Y, Cong X, Zhou Y, et al: Expression and clinical role of small glutaminerich tetratricopeptide repeat (TPR)-containing protein alpha (SGTA) as a novel cell cycle protein in NSCLC. J Cancer Res Clin Oncol 139: 1539-1549, 2013.

19. Weng MT, Tsao PN, Lin HL, Tung CC, Change MC, Chang YT, Wong JM and Wei SC: Hes1 increases the invasion ability of colorectal cancer cells via the STAT3-MMP14 pathway. PLoS One 10: e0144322, 2015.

20. Liu J, Lu WG, Ye F, Cheng XD, Hong D, Hu Y, Chen HZ and Xie X: Hes1/Hes5 gene inhibits differentiation via down-regulating Hash1 and promotes proliferation in cervical carcinoma cells. Int J Gynecol Cancer 20: 1109-1116, 2010.

21. Gao F, Huang W, Zhang Y, Tang S, Zheng L, Ma F, Wang Y, Tang $\mathrm{H}$ and $\mathrm{Li} \mathrm{X}$ : Hes1 promotes cell proliferation and migration by activating Bmi-1 and PTEN/Akt/GSK3 $\beta$ pathway in human colon cancer. Oncotarget 6: 38667-38680, 2015.

22. Ito T, Udaka N, Yazawa T, Okudela K, Hayashi H, Sudo T, Guillemot F, Kageyama R and Kitamura H: Basic helix-loop-helix transcription factors regulate the neuroendocrine differentiation of fetal mouse pulmonary epithelium. Development 127: 3913-3921, 2000

23. Kitamoto M, Nakanishi T, Kira S, Kawaguchi M, Nakashio R, Suemori S, Kajiyama G, Asahara T and Dohi K: The assessment of proliferating cell nuclear antigen immunohistochemical staining in small hepatocellular carcinoma and its relationship to histologic characteristics and prognosis. Cancer 72: 1859-1865, 1993.

24. Del Gobbo A, Pellegrinelli A, Gaudioso G, Castellani M, Zito Marino F, Franco R, Palleschi A, Nosotti M, Bosari S, Vaira V, et al: Analysis of NSCLC tumour heterogeneity, proliferative and 18F-FDG PET indices reveals Ki-67 prognostic role in adenocarcinomas. Histopathology 68: 746-751, 2015.

25. Kamakura S, Oishi K, Yoshimatsu T, Nakafuku M, Masuyama N and Gotoh Y: Hes binding to STAT3 mediates crosstalk between Notch and JAK-STAT signalling. Nat Cell Biol 6: 547-554, 2004. 